\title{
Face processing for security: a short review
}

Ion Marqués and Manuel Graña

\begin{abstract}
In this paper we give a fast fly over the face image preocessing issue, taking special care to highlight the security related applications. Face detection is the first step for the face recognition systems, posing its own challenges. Face recognition is essentially a classification problem, which can be a large multiclass problem. The emphasis in this paper is the of review the different computational approaches instead of the concrete applications.
\end{abstract}

\section{Introduction}

Face recognition is one of the most relevant applications of image analysis. It's a true challenge to build an automated system which equals human ability to recognize faces. There are many different industrial applications interested in it, most of them somehow related to security. Table 1 shows the most salient security applications. Engineering started to show interest in face recognition in the 1960's implementing semi-automatic systems $[2,7,11,8,9,10]$. In the 70's the most salient work was that of Kenade [25]. His face recognition program ran in a special purpose computer system. The algorithm extracted sixteen facial parameters automatically, and he demonstrated that better results were obtained when irrelevant features were not used. In the 1980's and 1990's there were a diversity of approaches, most of them continuing with previous tendencies. Some works tried to improve the methods used measuring subjective features like geometric measurement for eye spacing [34] others introduced novel computational methods, such as artificial neural networks [41]. The work of Turk and Pentland introducing eigenfaces for recognition [44] was a landmark for th development of the area. Their algorithm was able to locate, track and classify a subject's head. Since the 1990's, face recognition area has received a lot of attention, with a noticeable increase in the number of publications.

Computational Intelligence Group, Universidad del Pais Vasco www.ehu.es/ccwintco 
The structure of the paper is as follows: section 2 will be devoted to face detection, which is the previous step for robust face recognition that will be reviewed in section 3. For lack of space we will obviate the conclusions section.

\begin{tabular}{|c|c|}
\hline Areas & Applications \\
\hline Information Security & $\begin{array}{c}\text { Access security (OS, data bases) } \\
\text { Data privacy (e.g. medical records) } \\
\text { User authentication (trading, on line banking) }\end{array}$ \\
\hline Access management & $\begin{array}{c}\text { Secure access authentication (restricted facilities) } \\
\text { Permission based systems } \\
\text { Access log or audit trails }\end{array}$ \\
\hline Biometrics & $\begin{array}{c}\text { Person identification (national IDs, Passports, } \\
\text { voter registrations, driver licenses) } \\
\text { Automated identity verification (border controls) }\end{array}$ \\
\hline Law Enforcement & $\begin{array}{c}\text { Video surveillance } \\
\text { Suspect identification } \\
\text { Suspect tracking (investigation) } \\
\text { Simulated aging } \\
\text { Forensic Reconstruction of faces from remains }\end{array}$ \\
\hline Personal se & $\begin{array}{c}\text { Home video surveillance systems } \\
\text { Expression interpretation (driver monitoring system) }\end{array}$ \\
\hline
\end{tabular}

Table 1 Security applications of face recognition.

\section{Face detection}

Few applications of Face Recognition don't require face detection. Some criminal database images have face images already normalized to a given pose standard. However, the conventional input image of computer vision systems may contain many items and/or faces, either for recognition or tracking. In these cases face detection is mandatory, it is a required preprocessing of the images to determine, first, if there is a face in the image, and second, where it is located.

Knowledge-based face detection methods.

These systems try to capture our knowledge of the structure of faces, and translate it into a set of rules [27]. The big problem encountered by these methods is the difficulty of building the set of rules. It must be complete, consistent, and provide good detection performance. It is quite difficult to introduce noise and uncertainty robustness and to tune the system.

Another critical issue is to find invariant features for face detection like face-like image textures or the color of human skin. Some recent researches use more than one color model. For example, RGB and HSV may be used together successfully 
[45]. However, skin color can depend significantly on light conditions. Therefore, other methods, like local symmetry measures or structure and geometry, must be used in conjunction with the color models.

Template matching face detection methods

Template matching methods define a face image as a 3D function that can be compared to a standard template of all the faces [12]. The template can be defined on different features which can be defined independently, like the eyes, face contour, nose and mouth. Also a face model can be built by edges, or a silhouette. But these methods are most effective to find faces that are frontal and unoccluded with little variations in pose, scale and shape. Deformable templates have been proposed to deal with these problems [18].

Appearance-based face detection methods

Appearance-based methods rely on techniques from statistical analysis and machine learning to build the relevant feature extraction processes that will learn from a set of face images. These methods used include Eigenface-based methods [3], Distribution-based algorithms, Neural Networks [37, 39], Decision Trees [22], Support Vector Machines [35], Sparse Network of Winnows, Hidden Markov Models [32], Markov Random Fields or Inductive Learning methods.

Face tracking

Face tracking is essentially a motion estimation problem. Robust tracking have been proposed based on Kalman filters [16]. The state vector of a face includes the center position, size of the rectangle containing the face, the average color of the face area and the first image derivatives. The features are used in order, first a SSD algorithm works on the gray scale image, then the color information is used to confirm the detection. Robust optical-flow based recognition is in [21]

\section{Face recogntion}

The key face processing is face recognition for most applications [14]. It may consist in the authentication of a user, which a binary decision, or in the identification of a user which is a (large) multiclass problem. 


\subsection{Template matching face recognition methods}

Blanz and Vetter state in [6] that there are different ways of separating shape and orientation of a face in 3D models: To match feature vertexes to image positions and then interpolate deformations of the surface or to use restricted class-specific deformations, defined manually or automatically, from non textured or textured head scans. Separation between texture and illumination is achieved using models of illumination that consider illumination direction and intensity from Lambertian or non-Lambertian reflectance. The database of faces is obtained via 3D scans. The approach requires to manually define some feature points. The recognition process is done by building a 3D model of the subject. Then, this 3D model is compared with the stored patterns using two parameters -shape and texture. They have huge potential towards pose and illumination invariant face recognition. A high-order template based approach is presented in [49]. Incremental templates for video image face recognition are proposed in [17].

\subsection{Statistical learning for recognition algorithms}

Images of faces, represented as high-dimensional pixel arrays, often belong to a manifold of lower dimension. In statistical learning approaches, each image is viewed as a point (vector) in a $d$-dimensional space. The dimensionality of these data is too high. Therefore, the goal is to choose and apply the right statistical tool for extraction and analysis of the underlaying manifold. These tools must define the embedded face space in the image space and extract the basis functions from the face space. This would permit patterns belonging to different classes to occupy disjoint and compacted regions in the feature space. Consequently, we would be able to define a line, curve, plane or hyperplane that separates faces belonging to different classes. The classical approach applied Principal Component Analysis (PCA) for feature extraction [44], other approaches use the variations of the Linear Discriminant Analysis (LDA) [30, 53, 52, 29, 36, 47, 38, 13], or the Locality Preserving Projections (LPP) [19]. Other successful statistic tools include Bayesian networks [31], bi-dimensional regression [24], generative models [20], and ensemble-based and other boosting methods [30].

\subsection{Neural Network approaches}

Artificial Neural Networks (ANN) have succesfull in face recognition. They provide a training algorithm that eases the classifier building task. Some approaches like the Convolutional Neural Networks [28] try that the ANN learns also the feature extraction as well as the classification. 
Neural networks with Gabor filters

ANNs combined with Gabor filter [15, 1, 5, 40, 42, 48, 46, 50] assume a feature extraction pre-processing step based on Gabor filters. Every image is normalized in terms of contrast and illumination. Noise is reduce by a "fuzzily skewed" filter. Then, each image is processed through a banck of Gabor filters. For each face image, the outputs are 15 Gabor-images which record the variations measured by the Gabor filters. These images are the input to the ANN, a multilayer perceptron trained with the backprogation algorithm.

Neural networks and Hidden Markov Models

Hidden Markov Models (HMM) are a statistical tool originally developed for voice recognition and audio processing. They have been also used in conjunction with neural networks [4] for face recognition. They propose a pseudo 2D-HMM, defining superstates formed by states. The input of this 2D-HMM process is the output of the artificial neural network (ANN) applied to the input image to perform dimensionality reduction.

\subsection{Classifiers for face recognition}

Once the features are extracted and selected, the last step is to classify the image. Appearance-based face recognition algorithms use a wide variety of classification methods, and the literature has been very active in this area [51, 26]. An instance of a fuzzy discriminant has been recently proposed [33].

\section{Classifier combination}

Sometimes two or more classifiers are combined to achieve better results. The classifier combination problem can be defined as a problem of finding the combination function accepting M-dimensional score vectors from M-classifiers and outputting final classification scores. For lack of space we summarize in table 2 the combination schemes proposed in [43] and [23] that can be tested on face recognition problems.

\section{References}

1. Mohamed El Aroussi, Mohammed El Hassouni, Sanaa Ghouzali, Mohammed Rziza, and Driss Aboutajdine. Local appearance based face recognition method using block based steerable 


\begin{tabular}{|c|c|c|c|}
\hline Scheme & Architecture & Trainable & Info-level \\
\hline \hline Voting & Parallel & No & Abstract \\
\hline Sum, mean, median & Parallel & No & Confidence \\
\hline Product, min, max & Parallel & No & Confidence \\
\hline Generalized ensemble & Parallel & Yes & Confidence \\
\hline Adaptive weighting & Parallel & Yes & Confidence \\
\hline Stacking & Parallel & Yes & Confidence \\
\hline Borda count & Parallel & Yes & Rank \\
\hline Behavior Knowledge Space & Parallel & Yes & Abstract \\
\hline Logistic regression & Parallel & Yes & Rank \\
\hline Class set reduction & Parallel/Cascading & Yes & Rank \\
\hline Dempster-Shafer rules & Parallel & Yes & Rank \\
\hline Fuzzy integrals & Parallel & Yes & Confidence \\
\hline Mixture of Local Experts & Parallel & Yes & Confidence \\
\hline Hierarchical MLE & Hierarchical & Yes & Confidence \\
\hline Associative switch & Parallel & Yes & Abstract \\
\hline Random subspace & Parallel & Yes & Confidence \\
\hline Bagging & Parallel & Yes & Confidence \\
\hline Boosting & Hierarchical & Yes & Abstract \\
\hline Neural tree & Hierarchical & Yes & Confidence \\
\hline
\end{tabular}

Table 2 Classifier combination schemes

pyramid transform. Signal Processing, In Press, Corrected Proof:-, 2010.

2. Michael Ballantyne, Robert S. Boyer, and Larry Hines. Woody bledsoe: His life and legacy. AI Magazine, 17(1):7-20, 1996.

3. P.N. Belhumeur, J.P. Hespanha, and D.J. Kriegman. Eigenfaces vs. fisherfaces: Recognition using class specific linear projection. IEEE Transactions on Pattern Analysis and Machine Intelligence, 19(7):711-720, July 1997.

4. Vitoantonio Bevilacqua, Lucia Cariello, Gaetano Carro, Domenico Daleno, and Giuseppe Mastronardi. A face recognition system based on pseudo $2 \mathrm{~d} \mathrm{hmm}$ applied to neural network coefficient. Soft Computing - A Fusion of Foundations, Methodologies and Applications, 12(7):615-621, 2007.

5. Al-Amin Bhuiyan and Chang Hong Liu. On face recognition using gabor filters. In Proceedings of World Academy of Science, Engineering and Technology, volume 22, pages 51-56, 2007.

6. V. Blanz and T. Vetter. Face recognition based on fitting a $3 \mathrm{~d}$ morphable model. IEEE Transactions on Pattern Analysis and Machine Intelligence, 25(9):1063-1074, September 2003.

7. W. W. Bledsoe. The model method in facial recognition. Technical report pri 15, Panoramic Research, Inc., Palo Alto, California, 1964.

8. W. W. Bledsoe. Man-machine facial recognition: Report on a large-scale experiment. Technical report pri 22, Panoramic Research, Inc., Palo Alto, California, 1966.

9. W. W. Bledsoe. Some results on multicategory patten recognition. Journal of the Association for Computing Machinery, 13(2):304-316, 1966.

10. W. W. Bledsoe. Semiautomatic facial recognition. Technical report sri project 6693, Stanford Research Institute, Menlo Park, California, 1968.

11. W. W. Bledsoe and H. Chan. A man-machine facial recognition system-some preliminary results. Technical report pri 19a, Panoramic Research, Inc., Palo Alto, California, 1965.

12. R. Brunelli and T. Poggio. Face recognition: Features versus templates. IEEE Transactions on Pattern Analysis and Machine Intelligence, 15(10):1042-1052, October 1993.

13. Deng Cai, Xiaofei He, and Jiawei Han. Semi-supervised discriminant analysis. Computer Vision. IEEE 11th International Conference on Computer Vision, 14:1-7, 2007. 
14. Rama Chellappa, Pawan Sinha, and P. Jonathon Phillips. IEEE Computer, 43(2):46-55, 2010.

15. Weihong Deng, Jiani Hu, Jun Guo, Weidong Cai, and Dagan Feng. Emulating biological strategies for uncontrolled face recognition. Pattern Recognition, 43(6):2210 - 2223, 2010.

16. Kyunghan Baek et al. AI 2005, chapter Multiple Face Tracking Using Kalman Estimator Based Color SSD Algorithm, pages 1229-1232. LNAI 3809. Springer-Verlag Heidelberg, 2005.

17. Annalisa Franco, Dario Maio, and Davide Maltoni. Incremental template updating for face recognition in home environments. Pattern Recognition, 43(8):2891 - 2903, 2010.

18. P. Hallinan. A Deformable Model for Face Recognition Under Arbitrary Lighting Conditions. $\mathrm{PhD}$ thesis, Univesity of Harvard, 1995.

19. Xiaofei He and Partha Niyogi. Locality preserving projections. In Proceedings of the Conference on Advances in Nerual Information Processing Systems, 2003.

20. Guillaume Heusch and Sebastien Marcel. A novel statistical generative model dedicated to face recognition. Image and Vision Computing, 28(1):101 - 110, 2010.

21. Chao-Kuei Hsieh, Shang-Hong Lai, and Yung-Chang Chen. An optical flow-based approach to robust face recognition under expression variations. IEEE Transactions on Image Processing, 19(1)(1):233-240, 2010.

22. J. Huang, S. Gutta, and H. Wechsler. Detection of human faces using decision trees. In Proc. Second International Conf. Automatic Face and Gesture Recognition, pages 248-252, 1996.

23. A.K. Jain, R.P.W. Duin, and J. Mao. Statistical pattern recognition: A review. IEEE Transactions on Pattern Analysis and Machine Intelligence, 22(1):4-37, January 2000.

24. Sarvani Kare, Ashok Samal, and David Marx. Using bidimensional regression to assess face similarity. Machine Vision and Applications, 21(3):261-274, 2008.

25. Takeo Kenade. Picture Processing System by Computer Complex and Recognition of Human Faces. PhD thesis, Kyoto University, November 1973.

26. Sang-Ki Kim, Youn Jung Park, Kar-Ann Toh, and Sangyoun Lee. Svm-based feature extraction for face recognition. Pattern Recognition, 43(8):2871 - 2881, 2010.

27. Constantine Kotropoulos and Ioannis Pitas. Rule-based face detection in frontal views. In in Proc. of IEEE Int. Conf. on Acoustics, Speech and Signal Processing (ICASSP 97), vol. IV, pages 2537-2540, 1997.

28. Steve Lawrence, C. Lee Giles, Ah Chung Tsoi, and Andrew D. Back. Face recognition: A convolutional neural network approach. IEEE Transactions on Neural Networks, 8:98-113, 1997.

29. J. Lu, K.N. Plataniotis, and A.N. Venetsanopoulos. Face recognition using kernel linear discriminant algorithms. IEEE Trans. on Neural Networks, 14(1):117-126, January 2003.

30. J. Lu, K.N. Plataniotis, A.N. Venetsanopoulos, and S.Z. Li. Ensemble-based discriminant learning with boosting for face recognition. IEEE Transactions on Neural Networks, 17(1):166-178, January 2006

31. A.V. Nefian. Embedded bayesian networks for face recognition. In Proc. of the IEEE International Conference on Multimedia and Expo, volume 2, pages 133-136, Lusanne, Switzerland, August 2002.

32. A.V. Nefian and M.H. Hayes. Hidden markov models for face recognition. In Proc. of the IEEE International Conference on Acoustics, Speech, and Signal Processing, ICASSP'98, volume 5, pages 2721-2724, Washington, USA, May 1998.

33. Xiao ning Song, Yu jie Zheng, Xiao jun Wu, Xi bei Yang, and Jing yu Yang. A complete fuzzy discriminant analysis approach for face recognition. Applied Soft Computing, 10(1):208 $-214,2010$.

34. M. Nixon. Eye spacing measurement for facial recognition. Proceedings of the Society of Photo-Optical Instrument Engineers, SPIE, 575(37):279-285, August 1985.

35. Edgar Osuna, Robert Freund, and Federico Girosi. Training support vector machines: An application to face detection. Proceedings of the IEEE Conf. Computer Vision and Pattern Recognition, pages 130-136, June 1997.

36. Lishan Qiao, Songcan Chen, and Xiaoyang Tan. Sparsity preserving discriminant analysis for single training image face recognition. Pattern Recognition Letters, 31(5):422 - 429, 2010. 
37. Feraud Raphael, Bernier Olivier, and Collobert Daniel. A constrained generative model applied to face detection. Neural Processing Letters, 5(2):11-19, April 1997.

38. Chuan-Xian Ren and Dao-Qing Dai. Incremental learning of bidirectional principal components for face recognition. Pattern Recognition, 43(1):318 - 330, 2010.

39. Henry A. Rowley, Shumeet Baluja, and Takeo Kanade. Neural network-based face detection. IEEE trans. Pattern Analysis and Machine Intelligence, 20(1):23-38, January 1998.

40. Richa Singh, Mayank Vatsa, and Afzel Noore. Face recognition with disguise and single gallery images. Image and Vision Computing, 27(3):245 - 257, 2009. Special Issue on Multimodal Biometrics - Multimodal Biometrics Special Issue.

41. T. J. Stonham. Practical face recognition and verification with wisard. In Hadyn D. Ellis, editor, Aspects of face processing. Kluwer Academic Publishers, 1986.

42. Christian Tenllado, José Ignacio Gómez, Javier Setoain, Darío Mora, and Manuel Prieto. Improving face recognition by combination of natural and gabor faces. Pattern Recognition Letters, 31(11): 1453 - 1460, 2010.

43. S. Tulyakov. Review of classifier combination methods. Studies in Computational Intelligence (SCI), 90:361-386, 2008

44. M. Turk and A. Pentland. Eigenfaces for recognition. Journal of Cognitive Neurosicence, 3(1):71-86, 1991.

45. Kong Wan-zeng and Zhu Shan-an. Multi-face detection based on downsampling and modified subtractive clustering for color images. Journal of Zhejiang University SCIENCE A, 8(1):7278, January 2007.

46. Chenghua Xu, Stan Li, Tieniu Tan, and Long Quan. Automatic 3d face recognition from depth and intensity gabor features. Pattern Recognition, 42(9):1895 - 1905, 2009.

47. W.S. Yambor. Analysis of PCA-based and Fisher Discriminant-Based Image Recognition Algorithms. Technical report cs-00-103, Computer Science Department, Colorado State University, July 2000

48. Lei Yu, Zhongshi He, and Qi Cao. Gabor texture representation method for face recognition using the gamma and generalized gaussian models. Image and Vision Computing, 28(1):177 $-187,2010$.

49. Baochang Zhang, Yongsheng Gao, Sanqiang Zhao, and Jianzhuang Liu. Local derivative pattern versus local binary pattern: Face ecognition with high-order local pattern descriptor. IEEE Transactions on Image Processing, 19(2):533-544, 2010.

50. Sanqiang Zhao, Yongsheng Gao, and Baochang Zhang. Gabor feature constrained statistical model for efficient landmark localization and face recognition. Pattern Recognition Letters, 30(10):922 - 930, 2009.

51. W.Y. Zhao and R. Chellappa. Image-based face recognition: Issues and methods. In M. Dekker B. Javidi, editor, Image Recognition and Classification, pages 375-402. Marcel Dekker, Inc., 2002.

52. Dake Zhou and Zhenmin Tang. Kernel-based improved discriminant analysis and its application to face recognition. Soft Computing - A Fusion of Foundations, Methodologies and Applications, 14(2):103-111, 2009.

53. S. Zhou and R. Chellappa. Multiple-exemplar discriminant analysis for face recognition. In Proc. of the 17th International Conference on Pattern Recognition, ICPR'04, pages 191-194, Cambridge, UK, August 2004. 